\title{
Navigating Connectivity in Reduced Infrastructure Environments
}

\author{
Paul Schmitt \\ UC Santa Barbara \\ pschmitt@cs.ucsb.edu
}

\author{
Elizabeth Belding \\ UC Santa Barbara \\ ebelding@cs.ucsb.edu
}

\begin{abstract}
Modern communications systems rely on assumptions of centralized systems and underlying infrastructure that make them vulnerable in computing within limits scenarios. In this paper we offer case studies of issues facing connectivity in three locations in developing regions as a proxy for networks in such scenarios. We explore obstacles presented by such circumstances and future research directions for systems in resource-poor networking environments. We propose democratizing infrastructure deployment in a bottomup manner and re-thinking network architectures to be more decentralized with less reliance on centralized support systems.
\end{abstract}

\section{INTRODUCTION}

It is difficult to underestimate the need for communications systems. Connectivity has become so fundamental that the United Nations suggested in 2011 that internet access is a human right [9]:

Given that the Internet has become an indispensable tool for realizing a range of human rights, combating inequality, and accelerating development and human progress, ensuring universal access to the Internet should be a priority for all States. Each State should thus develop a concrete and effective policy, in consultation with individuals from all sections of society, including the private sector and relevant Government ministries, to make the Internet widely available, accessible and affordable to all segments of population.

Despite the critical need, modern networks have not been designed for graceful degradation in the face catastrophic or eventual collapse. Rather, global communications networks are largely hierarchical with mutual dependencies and assumptions that are simply untenable if we experience failures due to limits. In this work we focus on the use of wire-

Permission to make digital or hard copies of all or part of this work for personal or classroom use is granted without fee provided that copies are not made or distributed for profit or commercial advantage and that copies bear this notice and the full citation on the first page. Copyrights for components of this work owned by others than ACM must be honored. Abstracting with credit is permitted. To copy otherwise, or republish, to post on servers or to redistribute to lists, requires prior specific permission and/or a fee. Request permissions from permissions@ acm.org.

LIMITS '16, June 08-10, 2016, Irvine, CA, USA

(c) 2016 ACM. ISBN 978-1-4503-4260-5/16/06 . . $\$ 15.00$

DOI: http://dx.doi.org/10.1145/2926676.2926691 less technologies as we believe they are the most plausible solution for providing connectivity in resource-poor environments.

We draw upon our experiences with connectivity challenges in developing regions to argue for future research directions in wireless networking systems that can remain viable during collapse. We rely on our more than seven years of research on technology in developing regions and field work in more than seven sites to examine challenges to providing more universal internet access. We use three specific locations with differing levels of failure as real-world examples of what we anticipate networks will behave like in limited scenarios. Our case studies provide an entry point into our exploration of potential system solutions for providing connectivity in resource-poor environments as well as current barriers facing such systems. Based on this analysis, we highlight future research directions for the wireless networking community to address issues focused around reliance on centralized systems and strictly enforced top-down hierarchies.

\section{CASE STUDY LOCATIONS}

To discuss ramifications for communications networks in future realities where computing is governed by fundamental limits we must identify plausible limits scenarios. In this section we introduce the unique connectivity challenges faced by three locations at various stages of economic development. We believe these examples are able to serve as proxies for network conditions in limits scenarios, which we define to be due to infrastructure decline and failure due to catabolic collapse [5]. We summarize the salient features of each in Table 1.

Za'atari refugee camp, Jordan: Za'atari is the largest of four refugee camps in Jordan. Located in a previously rural desert region near the border with Syria, the camp was opened in July 2012 and its population quickly rose to 120,000 . It currently hosts about 80,000 residents in roughly 6 square kilometers. Given the rapid influx of people and the previously rural location, Za'atari provides an excellent example of overburdened cellular network infrastructure. We visited Za'atari and collected cellular measurements over the course of three days in January 2015.

Mobile service in the camp is offered by three carriers: Zain, Umniah, and Orange. Much of their infrastructure is located outside the camp and was initially built to serve the surrounding rural community that existed prior to the camp's development. We discussed carriers with camp residents and were told that Orange is popular in the more 


\begin{tabular}{|l|c|c|c|c|c|}
\hline Location & $\begin{array}{c}\text { Incumbent } \\
\text { Mobile Coverage }\end{array}$ & $\begin{array}{c}\text { Incumbent } \\
\text { Fixed Coverage }\end{array}$ & $\begin{array}{c}\text { User } \\
\text { Density }\end{array}$ & $\begin{array}{c}\text { User } \\
\text { Technologies }\end{array}$ & $\begin{array}{c}\text { User } \\
\text { Income }\end{array}$ \\
\hline Za'atari Camp, Jordan & yes & no $^{\dagger}$ & urban & Mobile, minimal fixed & $\$ \$$ \\
\hline San Cristóbal, Guatemala & partial & no & rural & Mobile & $\$$ \\
\hline Macha, Zambia & spotty & partial $^{\ddagger}$ & rural & Mobile, minimal fixed & $\$$ \\
\hline
\end{tabular}

$\dagger$ Terrestrial microwave link available to camp management. Residents do not have access.

* Two of three national commercial providers offer coverage in community.

¥ Backhaul link was extremely slow, unreliable, expensive, and available to only a very small subset of the population.

Table 1: Comparison of case study locations.

urban areas of Amman, while Zain is popular for rural customers. Umniah was generally not used by people with whom we spoke. Zain is the most popular carrier in the camp as SIM cards for the network are given to residents by camp administration as they first arrive and register at the camp. Residents of the camp are highly digitally-literate and expressed discontent with the limited connectivity options available throughout the camp. Locations in the camp with reduced camp management presence (i.e. residential areas rather than aid organization headquarters, schools, etc.) often have poor data connectivity such as EDGE or lack cellular data connectivity altogether. Through our analysis, we found extreme levels of radio resource congestion [15], particularly on the Zain network. Such congestion manifests in the delayed ability for phones to gain radio channels needed for voice/SMS or data sessions. During times of the day when the network was highly congested, we routinely observed GSM base stations forcing phones to wait up to 128 seconds before re-requesting radio channel access. It is evident that the infrastructure serving the camp has not been adequately scaled-up in response to the large increase in user demand. A potential cause for the lack of infrastructure augmentation by the commercial carriers has been the perceived temporary nature of the camp and generally negative sentiment toward refugees by Jordanian residents.

The camp is managed by the United Nations High Commissioner for Refugees (UNHCR), which largely operates out of a fenced-off portion of the camp known as "base-camp." UNHCR uses a fixed terrestrial microwave link to a nearby town in order to obtain internet connectivity. The link is shared throughout base-camp via LAN and WiFi. Residents are not given access to this link. This is due to UNHCR's organizational policy of providing all services to refugees free of charge. If the internet link was made available to residents, the monthly cost would be too large for UNHCR to pay. The long-standing free services policy has led to political unrest in the camp as Syrian refugees come from a middle income country and many residents expect high quality connectivity.

San Cristóbal Verapaz, Guatemala: Guatemala is one of the poorest countries in Latin America, with a GDP per capita of around $\$ 3,512$. Despite this, mobile carriers within Guatemala have invested heavily in cellular infrastructure, providing connectivity to a large portion of the country. We conducted field work in the rural city of San Cristóbal Verapaz over eight days in January 2015 in order to assess the coverage and quality of cellular connectivity in the area. As in Za'atari, we captured and analyzed cellular broadcast messages in order to infer the activity as well assess the ability of the existing cellular infrastructure to meet local demand.

Three mobile carriers offer service in Guatemala, all based on GSM: Tigo, Claro, and Movistar. Generally, in rural areas such as San Cristóbal, only Tigo and Movistar offer coverage as Claro's network deployment is focused on providing $3 \mathrm{G}$ coverage to more densely populated areas. In our measurement campaign, we found that the cellular infrastructure serving the city experienced frequent radio resource congestion. This was evidenced by base stations sending rejection messages in response to client requests for dedicated communications channels, similar to our traces collected in the Za'atari refugee camp, though on a less-severe scale. Such behavior strongly indicates oversubscribed infrastructure and results in delays and disconnections for users attempting to use the networks.

Macha, Zambia: Macha is a Sub-Saharan Africa village where residents are scattered over a large area, resulting in a low population density typical of rural areas in the region. The consequence of such sparse populations is a general lack of network infrastructure as well as a lack of motivation for service providers to expand coverage as revenues from such a population would likely be too low to justify the expenditure. As such, Macha has limited network connectivity. The local economy is based around maize farming and most residents have limited digital literacy. The local population is roughly 135,000; however, it is rather sparse as the village has a radius of around $35 \mathrm{~km}$ [19], making the population density 25 per $\mathrm{km}^{2}$.

Macha is somewhat unique in that a local non-governmental organization (NGO), along with networking researchers, built a community network that shares a $2 \mathrm{Mbps}$ internet access link across a small area of the community. The network is delivered using 802.11 devices acting as point-to-point links, hotspots, and layer-2 bridges. Public internet access is gained through an internet café ${ }^{1}$. Commercial cellular carrier coverage also exists in the area; however, connectivity is unpredictable. We visited Macha on numerous research trips to characterize network conditions as well as to trial systems designed for the local connectivity limitations. In prior work, we trialed a local cellular network based on OpenBTS [1] in Macha that provided basic GSM service to local users $[20]$.

\subsection{Correlations with limits scenarios}

A question we must consider is whether these locations provide appropriate representations of networks in fundamental limits scenarios. Of course, we are unable to predict which failure models (e.g. peak oil, climate change, etc.) will affect networks and locations around the world. Chen [4] dis-

\footnotetext{
${ }^{1}$ To the best of our knowledge, the Macha network is now defunct as the local partner no longer maintains a presence in the community. The network was operational for 4 years, ending in roughly 2012 .
} 
cusses similarities between information and communications technologies for development (ICTD) and limits scenarios and uses a failure model based around catabolic collapse [5] as an example. In such a scenario, network infrastructure will eventually deteriorate and / or fail due to inadequate maintenance.

The descent of networks in collapse scenarios are likely to be non-uniform [13]. We argue that our case study locations offer examples of networks experiencing different rates of descent and degrowth. Za'atari shows us what we may expect to see in cases of catastrophic failure or cases of mass forced migration to previously uninhabited locations. Network conditions in San Cristóbal can give insights into earlystage collapse as the networks are largely operational, yet are not able to fully service user demand. Lastly, Macha provides an analogue to late-stage collapse scenarios where connectivity is poor and prohibitively expensive. The result is that the local user population essentially is unable to use the infrastructure that exists. Connectivity in Macha is also greatly affected by non-networking infrastructure, such as the electrical grid, which is unpredictable and often fails. This dependence on external systems illustrates the frailty of a network in such an environment.

\section{BARRIERS}

Across all three of our case study locations we witness various levels of network failure due to existing service providers that either choose not to or are otherwise unable to meet the local demand. In this section we enumerate some of the current challenges that arise when attempting to improve connectivity in resource-poor contexts.

\subsection{Infrastructure hierarchies and oligarchies}

Modern internet and cellular networks are deeply hierarchical and controlled by relatively few parties. The inertia of this reality makes fundamentally altering the delivery of connectivity challenging, particularly for those that are not a part of the controlling organizations.

The reasons behind the hierarchical nature of networks are partly a relic of prior networking design practices. For example, cellular core networks based on GSM standards contain many user-facing base stations that are controlled by fewer base station controllers which, in turn, are serviced by even fewer core entities such as subscriber registries and gateways [3]. These hierarchies were designed when computing infrastructure was focused around the use of mainframes and performance predictability was controlled by having centralized systems doing the majority of processing. Newer standards, such as LTE, are the industry response to the explosion of data usage over cellular networks, which has uncovered inefficiencies in the hierarchical topologies. For instance, most carriers operate relatively few IP gateways, where user data sessions ingress and egress onto the routable internet. Those few gateways result in poor user localization from an internet perspective [14], resulting in users often connecting to distant servers rather than physically nearby ones. LTE network design principles 'flatten' the hierarchy by de-tunneling user data traffic into IP traffic near the edge in order to reduce latencies imposed by previous hierarchical designs.

In theory, this flattening should enable innovation at the local level as base stations can offer service in a more decentralized manner. However, the incumbent cellular carriers do not allow infrastructure deployed by outside parties to connect into the proprietary core. This oligarchic nature of cellular infrastructures means that it is nearly impossible for those in the 'bottom of the pyramid' to add or augment connectivity, even at a local level. Cellular networks are closed systems, with no requirement to interface with other networks.

\subsection{Policies and regulations}

A more subtle challenge facing widespread cellular and broadband connectivity is due to wireless spectrum regulations that are enacted at the national-scale. For instance, cellular technologies operate in licensed spectrum, which means that service providers obtain exclusive rights from governments to deploy infrastructure that operates within set frequency ranges. These agreements are needed to protect the licensees (providers) from wireless interference that can degrade services and cause communication failures. In areas with dense populations that are economically viable for the providers to operate within, exclusive use of spectrum is crucial for providing predictable quality wireless connectivity. For example, the entire $900 \mathrm{MHz}$ frequency range used by GSM includes a total of 125 non-overlapping wireless channels. As GSM base stations can have a range of up to $35 \mathrm{~km}$, providers in dense areas, such as urban cities, clearly have a need for strict spectrum licensing enforcement; without it they would quickly exhaust the available frequencies and interference due to frequency re-use would degrade performance.

However, the national scale of spectrum licensing rights has an unintended consequence for providing coverage in rural areas. There is currently no legal means for rural communities to deploy local-scale wireless infrastructure that operates in licensed bands, despite the fact that in many rural locations the licensed frequency spectrum is left relatively unused. Additionally, spectrum regulation has been slow to evolve compared with the rapid advancement of wireless technologies. Whereas the traditional policies and regulations are inflexible, new radio technologies, such as software defined radios (SDRs), open the possibility of agile frequency use. Using SDRs, we can now sense a given frequency and determine whether it is occupied by a carrier. We can also flexibly operate wireless communication channels within broad ranges of frequency spectrum. Spectrum regulators have begun to react to the technological advancements by proposing new spectrum occupancy and licensing models such as licensed shared access (LSA) and authorized shared access (ASA). These new models allow for spectrum to be used by more than one entity within a regulatory environment. Trials have been conducted using the new sharing models and show promise [11]. Essentially, primary licensees continue to have exclusive rights to spectrum they have purchased; however, secondary providers can operate in licensed spectrum in locations where the primary licensee does not use it. LSA and ASA represent major steps forward for small-scale wireless system operation. Unfortunately, the pace at which new spectrum licensing models are actually put into place is dictated by the speed of governments.

\subsection{Barriers in limits scenarios}

How do the current challenges of infrastructure and the regulatory environment translate in a collapse scenario? Do these barriers persist? In the case of infrastructure hierar- 
chies and the companies that control them, we believe the barriers will continue if network operators do not plan for the possibility of decline or collapse. The closed, proprietary nature of core networks makes them rigid and unable, in their current state, to alter the way services are delivered or to use alternate infrastructures. To prepare for decline, incumbent carriers should investigate methods and protocols to enable connectivity via networks in which they do not own and control $100 \%$ of the infrastructure.

Challenges related to spectrum policies and regulations seem likely to be reduced in the future. The advancement of software-defined radios and proposed sharing models point to a future where dynamic spectrum access is possible. Additionally, in the case of a global decline or collapse, we imagine spectrum will become less occupied and the need for regulation may also be diminished. However, current policies hamper innovation and exploration of new wireless systems. In order to design novel solutions, we must be able to test and operate them in real-world environments.

\section{RESEARCH AGENDA}

Residents of limited network environments require new solutions that can provide reliable cellular and broadband internet access. Systems and technologies that maximize coverage and per-user bandwidth while minimizing cost are desperately needed. We believe the most cost-effective, plausible solutions to providing such connectivity are based on the use of cellular technologies for internet backhaul as the majority of users we have interacted with have access to cellular devices, whereas PCs are much more rare. In this section we argue for systems research with respect to the obstacles we have observed. We also include what we see as open questions in each area.

\subsection{User-extensible, bottom-up infrastructures}

Reliance on centralized, top-down infrastructure is untenable in a collapse scenario. Therefore, those that best know the critical networking needs at a local level must have the ability to build and extend connectivity infrastructure on an ad-hoc basis. Incumbent carriers can enable such bottom-up infrastructure by offering publicly available interfaces into their core networks. Regulatory agencies must also shift toward dynamic use of wireless spectrum in order to ease the policy restraints facing many solutions.

\subsubsection{Local cellular networks}

In all three example locations, locally-deployable cellular infrastructures present an interesting potential direction. Recent work has deployed local cellular networks [7, 6, 20] in remote areas without existing service. Successful deployments by nonprofit [2] and community organizations show that it is technologically and economically sustainable for community groups to deploy and operate their own "popup' cellular networks, which can quickly expand and shrink in concert with demand. Further, through user traffic analysis, we have found strong indications of locality of interest in communication in developing regions [8, 17, 21], making the case for locally operated small-scale cellular networks in which users can SMS or call each other without needing any connection to the internet or the public switched telephone network (PSTN). Such operation is critical in developing regions, as internet backhaul links can often unexpectedly fail, or be prohibitively expensive.
The Za'atari refugee camp is an interesting case as Jordan has a more developed economy compared with Zambia and Guatemala. However, the existing carrier networks operating in Za'atari are overwhelmed by the current demand placed on them. We witnessed extreme levels of radio resource congestion on cellular base stations in the camp, as well as evidence that the camp-serving infrastructure was actually located in a community roughly $10 \mathrm{~km}$ away from the camp. The issue that arises when a large concentration of users is served by far-away infrastructure is that user devices will not negotiate higher data rates because coding schemes are determined based on the signal quality between the base station and the mobile device, which is heavily influenced by distance between the two. During our field work, we collected cellular measurements that allowed us to analyze success and failure in obtaining private communication channels between mobile devices and base stations. We observed extreme failure rates. For instance, we see over $30 \%$ failure to obtain radio resources on the Zain network, versus $0.03 \%$ failure on AT\&T in Santa Barbara. The population of users within the camp have simply overwhelmed the existing carrier infrastructure. Users report failures throughout the day and general difficulty to connect to the internet [12]. This was verified by our trace analysis.

The research challenge facing local cellular solutions is the presence of existing service and the regulations governing spectrum occupancy for a given area. Any local cellular network, if theoretically allowed to legally operate, must coexist peacefully with incumbent carriers. We have designed an initial system, named HybridCell [16], based on our experiences in Za'atari. HybridCell uses a local cellular network installation as well as an application designed for rooted Android smartphones to programmatically duty cycle between the commercial and the local network without requiring user intervention. The duration of time spent on the local network versus the commercial networks is dictated by the estimated congestion of the commercial networks (i.e. if the commercial networks are failing at a high rate, more time is spent on the local network, and vice versa). We use SDRs to sense primary licensee base stations' frequencies as well as technology in use. Our analysis has shown that users would receive a higher likelihood of obtaining cellular connectivity by using HybridCell in areas with overburdened infrastructure by dynamically shifting some of the user burden off of the commercial network during congestion events. Another challenge faced by local cellular infrastructure is the difficulty in obtaining interconnection with other networks [7]. For local cellular to succeed, networks must offer transport for traffic originating on other networks, similar to the 3tiered model used by internet service providers.

Za'atari residents also expressed a need for internet connectivity in multiple desktop computer labs scattered throughout the camp. Whereas the camp administrators have a terrestrial microwave backhaul providing a fixed internet link, the community centers and schools throughout the camp use $3 \mathrm{G}$ dongles connected to $\mathrm{WiFi}$ access points in order to connect to the internet. This solution increases the complexity and rate of disconnections in the schools, as the dongles often overheat and fail or run out of credits. Much of the functionality could be achieved through the use of a campwide WiFi-based LAN. Schools could pool their resources and share a common internet backhaul rather than doing so on an ad-hoc basis. 
Our analysis of the San Cristóbal area was focused on the existing commercial cellular providers operating in the area and the resulting network quality / congestion. We found that, of the three carriers that provide service in Guatemala, only two had base stations in San Cristóbal, with Claro service not present in the community. Additionally, we found that the two providers that do offer service in the area, Tigo and Movistar, have infrastructure that appears to be overburdened by users. We infer this through the analysis of cellular broadcast messages, particularly messages used to indicate the success or failure of private communication channels needed for voice/SMS or data sessions. We found failure rates 21 times higher than those experienced on U.S. carrier networks.

Our findings lead us to believe that San Cristóbal could benefit from a local cellular network deployment. We observed that users connected to the same base stations as our monitoring devices requested voice and SMS services at a higher rate than similar users on U.S. networks (i.e. a higher percentage of U.S. requests were for data rather than voice ( SMS). This provides another strong argument for the use of local cellular networks, as voice and SMS services are straightforward to enable using basic GSM. San Cristóbal includes a regional hospital which attracts locals as well as people from nearby communities. A network of local cellular networks, with neighboring communities connected via directional WiFi links, could provide an essential lifeline between patients and families that must remain at home in order to work. The spectrum regulations currently in place in Guatemala bar the operation of a local cellular network despite the fact that the community has large amounts of unused spectrum.

As stated earlier, we deployed a trial local cellular network in Macha in 2012 [20]. The network was initially built using two OpenBTS base stations and was intended to serve a small number of local users for research and testing purposes. The network also included traditional computers which were used by a school for IT training and an internet café. The available frequency range was scanned and the local cellular network operated in unused $1800 \mathrm{MHz}$ spectrum. The users were satisfied with the network service and were eager to expand the user base in order to be able to connect to more friends and neighbors. Unfortunately, our local partner ceased operations which effectively ended the network trial. However, an important fact is that the local cellular network, which could have potentially provided a useful service to a community with few other options, operated without a license in licensed spectrum. At any point government regulators could have shut the deployment down. We believe that there must be consideration of a compromise for non-interfering systems that provide a social good.

Questions:

- Can locally-owned and built infrastructure offer truly seamless connectivity between commercial and local carriers?

- Can cellular networks be re-architected to resemble internet service providers (e.g. tiers 1 - 3) in terms of transit and peering?

- What must be changed to have universal interconnect agreements between all carriers?

\subsubsection{Unlicensed cellular}

Another promising research direction is through the explo-

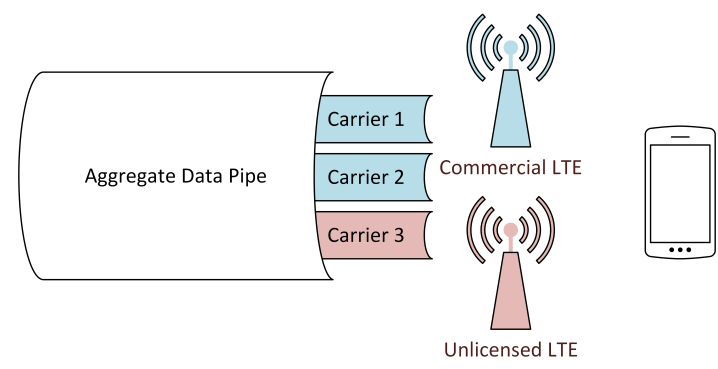

Figure 1: Carrier aggregation overview. Heterogeneous LTE carriers are logically bonded to form a large data pipe.

ration of cellular connectivity within unlicensed frequency bands. One of the major barriers to providing local cellular networks is that those technologies occupy licensed spectrum. Recently, the telecommunications industry has begun developing hardware that operates in unlicensed ISM bands (e.g. WiFi frequencies). Carriers take advantage of a new mechanism available in the LTE specification known as carrier aggregation, where multiple data tunnels are logically bound to appear as a single link on the mobile device. As shown in Figure 1, a user device utilizing carrier aggregation can connect to multiple LTE base stations, in both licensed cellular bands and unlicensed bands, and bind all of the available carrier channels logically as one large channel, thus increasing available bandwidth over heterogeneous networks. Unfortunately, the primary carriers that are driving the research in this space have expressed the intent to only use carrier-approved base stations that operate in unlicensed spectrum so that they have full control over the quality of service and deployment of unlicensed cellular. Commercial carriers have also begun exploring the use of the newly available $3.5 \mathrm{GHz}$ band in the United States. We believe that unlicensed cellular should instead focus on bands that are globally available to enable economies of scale for wireless chipset producers.

We believe this top-down, carrier-controlled model is shortsighted. Mobile traffic is shifting toward the use of the IP multimedia subsystem (IMS) architecture, meaning call services such as voice and SMS are placed over packet-switched networks rather than the traditional circuit-switched networks. This transition, as well as the rise in processing power of mobile devices, provides the opportunity for us to re-think mobile connectivity. Today, more and more smartphones are capable of operating their own voice-over-IP (VoIP) software directly on the device. This means that the voice or SMS traffic exiting the phone can simply be data packets and any internet connection will suffice for connectivity. In fact, voice-over-LTE (VoLTE) is a new standard that is beginning to be rolled out by some carriers and is often touted as an upgrade to 'high-definition' voice as the codecs used can take advantage of large data bitrates for high fidelity audio.

Rather than allowing carriers to fully control deployments, we argue that unlicensed cellular should adopt the model of WiFi. The proliferation of $\mathrm{WiFi}$ was made possible by the abundance of affordable, consumer-grade hardware that made it easy to install an access point anywhere there was an internet connection. We envision the same hardware (e.g. commodity home routers) could be outfitted with an LTE baseband chipset enabling them to advertise both WiFi and LTE channels. Phones with this capability could simply 
treat the additional LTE channel as a supplemental data link using carrier aggregation.

Commercial carriers would benefit from this scenario as data usage would be offloaded from their networks in the same way it is currently offloaded onto WiFi networks. If billing and accounting are required, the commercial carriers could open an interface in which phones could connect back to the core. If carriers require a particular quality of service metric, the internet backhaul link can be monitored and latency-sensitive services (e.g. voice) over the LTE access point can be programmatically disabled in cases of extremely poor internet connectivity. Lastly, users could experience improved energy consumption as they could choose to disable the WiFi radio and only use the cellular baseband radio on their phones, as $\mathrm{WiFi}$ radios are typically one of the top energy consumers in phones.

\section{Questions:}

- How can a WiFi-like model be adopted for cellular?

- Can service guarantees be achieved for decentralized, open-access cellular?

- What must occur to treat cellular as a utility-like service where unlicensed cellular base stations that offer service are akin to solar cells that feed power back into the electrical grid?

\subsection{Bring your own infrastructure (BYOI)}

While bottom-up infrastructures would provide a great benefit in resource-poor environments, research should also focus on fundamental connectivity alternatives. As it stands today, network infrastructure is typically highly specialized, expensive equipment that is manufactured and sold by very few companies. The unseen superstructures (e.g. manufacturing, materials, distribution, power, etc.) that make up and support this system cannot be relied upon in a scenario where infrastructures deteriorate and collapse due to exogenous limits. Our experiences with forcibly displaced refugees lead us to believe that we must reconsider the equipment and assumptions we currently use to deploy network infrastructure. We must research and design wireless systems that utilize equipment that we can expect to be readily available and not dependent on always-on grid power (e.g. smartphones). Ideally, smartphones could act as communication infrastructure both on an ad-hoc basis with no need for global connectivity, as well as in circumstances where they can be globally-connected. BYOI research would mean infrastructure migrates with people in their pockets.

LTE-Direct: Infrastructure-less cellular communication has been one of the thrusts of the next generation LTEAdvanced standards. Using LTE-Direct, user devices will be able to send SMSs and place voice calls without the use of a cellular base station, provided the users are near one another. Prior research in such networks has often focused on the challenges faced by carriers using direct communications [10]. We believe that LTE-Direct can provide a foundation for BYOI networking. However, to be appropriate for collapse scenarios, research must be done with no assumption of a centralized carrier control or presence.

\section{Questions:}

- Can phones be re-purposed to act as cellular infrastructure?

- How can existing and upcoming standards (e.g. LTEDirect) be leveraged to use phones as both end-user devices and backhauls for other users?

\subsection{Networks of networks}

Modern communications networks, and particularly cellular networks, are hierarchical and unserviceable by regular people. These characteristics render them incapable of being "collapse-compliant" [18]. If we aim to provide agile, highquality connectivity when facing limits, we must begin to rearchitect networks to be capable of functioning in the face of collapse. The internet was originally envisioned as a decentralized network of networks. While it can be argued that it remains such today, many critical services are relatively centralized and user connectivity relies on underlying assumptions of global connectivity. To make connectivity feasible in cases with those assumptions removed, researchers must return to network of networks design principles. Increased localization and decentralization of connectivity is critical for networks to survive failure due to limits.

\section{Questions:}

- How can centralized internet services be designed for local, offline usage?

- How must network topologies be altered to be robust to external failure?

\section{CONCLUSIONS}

Present-day networks are ill-prepared for collapse and will be unsustainable if we continue our current trajectories. To design for collapse, we must re-think architectures and work without assumptions that currently govern communications networks. Recent and upcoming standards have the chance to improve connectivity in extremely challenging environments, which could ultimately impact billions of people.

Through real-world experiences, this paper explores network collapse and potential directions for research to provide connectivity in resource-deprived areas. The wireless networking research community has the opportunity to help shape future connectivity models. To be viable, we must begin researching and designing systems that operate independently at a local scale, without reliance on global connectivity.

\section{ACKNOWLEDGEMENTS}

This work was funded through NSF Network Science and Engineering (NetSE) Award CNS-1064821 and NSF Catalyzing New International Collaborations (CNIC) Award IIA-1427873.

\section{REFERENCES}

[1] http://www.openbts.org. [Online; accessed: 2016-04-01].

[2] So much going on! http: //rhizomatica.org/2015/01/14/so-much-going-on/. [Online; accessed: 2016-04-8].

[3] 3GPP. Network architecture. TS 23.002, 3rd Generation Partnership Project (3GPP), Sept. 2008.

[4] J. Chen. Computing within limits and ICTD. First Monday, 20(8), 2015.

[5] J. M. Greer. How civilizations fall: A theory of catabolic collapse. http://ecoshock.org/transcripts/greer_on_collapse.pdf, 2005. [Online; accessed 20-April-2016].

[6] K. Heimerl and E. Brewer. The village base station. In NSDR, San Francisco, CA, USA, June 2010. 
[7] K. Heimerl, S. Hasan, K. Ali, E. Brewer, and T. Parikh. Local, sustainable, small-scale cellular networks. In ICTD '13, Cape Town, South Africa, Dec. 2013.

[8] D. L. Johnson, E. M. Belding, and G. van Stam. Network traffic locality in a rural african village. In ICTD '12, Atlanta, Georgia, USA, Mar. 2012.

[9] F. La Rue. Report of the special rapporteur on the promotion and protection of the right to freedom of opinion and expression. United Nations, May 2011.

[10] L. Lei, Z. Zhong, C. Lin, and X. Shen. Operator controlled device-to-device communications in LTE-advanced networks. IEEE Wireless Communications, 19(3):96-104, June 2012.

[11] M. Palola, M. Matinmikko, J. Prokkola, M. Mustonen, M. Heikkilä, T. Kippola, S. Yrjölä, V. Hartikainen, L. Tudose, A. Kivinen, J. Paavola, and K. Heiska. Live field trial of licensed shared access (LSA) concept using LTE network in $2.3 \mathrm{GHz}$ band. In Dynamic Spectrum Access Networks (DYSPAN), Mclean, VA, USA, Apr. 2014.

[12] M. Pizzi. Logging on in Za'atari: Part I. http://www.smex.org/logging-on-in-zaatari-part-i/. [Online; accessed: 2016-04-25].

[13] B. Raghavan and J. Ma. Networking in the long emergency. In 2nd ACM SIGCOMM workshop on Green networking, Toronto, ON, Canada, Aug. 2011.

[14] J. P. Rula and F. E. Bustamante. Behind the curtain:
Cellular DNS and content replica selection. In $I M C$ '14, Vancouver, BC, Canada, Nov. 2014.

[15] P. Schmitt, D. Iland, E. Belding, B. Tomaszewski, Y. Xu, and C. Maitland. Community-level access divides: A refugee camp case study. In ICTD '16, Ann Arbor, MI, USA, June 2016.

[16] P. Schmitt, D. Iland, M. Zheleva, and E. Belding. HybridCell: Cellular connectivity on the fringes with demand-driven local cells. In IEEE INFOCOM '16, San Francisco, CA, USA, Apr. 2016.

[17] P. Schmitt, R. Raghavendra, and E. Belding. Internet media upload caching for poorly-connected regions. In ACM DEV, London, UK, Dec. 2015.

[18] B. Tomlinson, M. Silberman, D. Patterson, Y. Pan, and E. Blevis. Collapse informatics: augmenting the sustainability \& ICT4D discourse in HCI. In CHI '12, May 2012.

[19] Wikipedia. Macha, Zambia - Wikipedia, the free encyclopedia. http://en.wikipedia.org/wiki/Macha,_Zambia, 2014. [Online; accessed 4-April-2016].

[20] M. Zheleva, A. Paul, D. L. Johnson, and E. Belding. Kwiizya: Local Cellular Network Services in Remote Areas. In MobiSys '13, Taipei, Taiwan, 2013.

[21] M. Zheleva, P. Schmitt, M. Vigil, and E. Belding. Bringing visibility to rural users in Cote d'Ivoire. In ICTD '13, Cape Town, South Africa, Dec. 2013. 